\title{
On kinematics of very steep waves
}

\author{
L. Shemer \\ School of Mechanical Engineering, Tel-Aviv University, Tel-Aviv, Israel \\ Correspondence to: L. Shemer (shemer@eng.tau.ac.il)
}

Received: 9 April 2013 - Published in Nat. Hazards Earth Syst. Sci. Discuss.: 23 April 2013

Revised: 26 June 2013 - Accepted: 13 July 2013 - Published: 22 August 2013

\begin{abstract}
Experiments on extremely steep deterministic waves generated in a large wave tank by focusing of a broadbanded wave train serve as a motivation for the theoretical analysis of the conditions leading to wave breaking. Particular attention is given to the crest of the steepest wave where both the horizontal velocity and the vertical acceleration attain their maxima. Analysis is carried out up to the third order in wave steepness. The apparent, Eulerian and Lagrangian accelerations are computed for wave parameters observed in experiments. It is demonstrated that for a wave group with a wide spectrum, the crest propagation velocity differs significantly from both the phase and the group velocities of the peak wave. Conclusions are drawn regarding the applicability of various criteria for wave breaking.
\end{abstract}

\section{Introduction}

Three main factors determine the wave energy balance: input due to the action of wind, wave energy dissipation, and nonlinear interactions among waves. The nonlinear interactions among waves are fairly well understood; the interaction between wind and waves can be modelled effectively. In spite of the fact that breaking constitutes the dominant wave energy damping mechanism, it is not yet understood properly, so that the wave breaking constitutes one of the most challenging problems in physical oceanography.

During the wave breaking process, a substantial part of the wave energy is dissipated, mostly to turbulent kinetic energy of water velocity fluctuations and eventually to heat. The detailed mechanism of wave breaking and the exact conditions required for waves to break remain largely unknown. The breaking criteria suggested over the years may be divided in a broad sense into three types: geometric, kinematic and dynamic (Gemmrich, 2005; Babanin, 2011). A num- ber of geometric criteria related to the wave shape on the verge of breaking were suggested, such as maximum steepness $(a k \approx 0.443$ for Stokes wave, where $a$ and $k$ are the amplitude and the wave number, respectively), wave asymmetry in either horizontal or vertical direction, as well as maximum instantaneous surface slope (Longuet-Higgins and Fox, 1977). The kinematic criteria for wave breaking predict that the wave breaks if the orbital velocity at its crest exceeds either the phase or the group velocity of the wave, although recent experiments by Grue and Jensen (2012) seem to demonstrate that the maximum velocity at the crest of breaking wave does not exceed significantly $0.4 c$, where the wave phase velocity $c$ is computed taking into account nonlinear and current effects. Phillips (1958) offered a dynamic breaking criterion arguing that since for gravity water waves the gravity is the only restoring force, the maximum possible negative value of the vertical acceleration at the free surface $a_{\mathrm{v}}=-g$. Although straightforward and physically convincing, this criterion remains neither experimentally nor numerically supported. Longuet-Higgins (1985) noticed that the maximum negative value of the vertical acceleration in the steepest Stokes wave where a $120^{\circ}$ corner flow exists at the crest is only $a_{\mathrm{v}}=-g / 2$. In some field experiments the measured values close to $a_{\mathrm{V}}=-g / 2$ were reported, but most experiments yield wave breaking at downward acceleration values less than $g / 2$ (Jensen et al., 2007).

It should be stressed that when experimental verification of the dynamic criterion by Phillips (1958) is considered, distinction should be made between the "apparent" acceleration defined by Longuet-Higgins (1985) as $\partial^{2} \zeta / \partial t^{2}$, the Eulerian vertical acceleration $a_{\mathrm{E}}=\partial w / \partial t$, and the Lagrangian acceleration of the fluid particle, $a_{\mathrm{L}}$. While in linear approximation all these accelerations are identical, they can differ essentially when nonlinear terms are accounted for. For unidirectional waves, the velocity $\boldsymbol{v}=(u, w)$ at the free surface 
$\zeta=\zeta(x, t)$ is related to $\zeta$ by the kinematic boundary condition

$w=\partial \zeta / \partial t+\cdot u \cdot \partial \zeta / \partial x$ at $z=\zeta(x, y)$.

It is evident from Eq. (1) that at higher orders the Eulerian vertical acceleration $a_{\mathrm{E}} \neq \partial^{2} \zeta / \partial t^{2}$. The "apparent" acceleration $\partial^{2} \zeta / \partial t^{2}$ can be measured relatively simply; the experimental determination of $a_{\mathrm{E}}$ however is significantly more complicated since it requires simultaneous measurements of the horizontal component of the instantaneous surface velocity and of the surface slope. The Phillips dynamic criterion is based on the Lagrangian vertical acceleration $a_{\mathrm{L}}$, a quantity that is even more difficult to measure. In this respect it should be noted that the contribution of higher order (bound) waves is essential not only to the material acceleration of the water particle, but also to the velocity components (Johannessen, 2010).

The present study that considers broad-banded wave groups was motivated by an observation made during experiments on unidirectional random water waves in a large wave tank (Shemer et al., 2010). In the course of that study, it was noticed that for a fixed wave peak frequency and steepness, maximum accelerations and velocities of the wavemaker piston required for excitation of the prescribed wave field depend on the spectral shape and increase strongly as the spectrum gets wider. This observation led to a plausible assumption that while the negative vertical acceleration remains significantly below gravitational acceleration $g$ even for the steepest possible Stokes wave, the situation may differ for a wave field with a wider spectrum. The horizontal velocities at crests of steep waves in a broad-banded wave field may also exceed significantly estimates based on a single dominant mode. Moreover, both the vertical accelerations and the horizontal velocities of fluid particles at the free surface of extreme waves that are essentially nonlinear differ significantly from those predicted by the linear wave theory. Previous measurements in a large wave tank (Shemer et al., 2007) provided records of deterministic steep focused waves with a wide spectrum under controlled conditions. In these experiments, a dispersive deep or intermediate-depth wave train was generated by a wavemaker in such a way that it evolved into a single steep wave at a prescribed location. In some experiments the steep wave underwent breaking close to the prescribed focusing location, while for other wave parameters no breaking wave observed. Since direct measurements of the horizontal velocities and of the horizontal and vertical acceleration components of water particle at the crest of the steepest wave are extremely difficult to perform, these quantities have to be computed.

Several possible approaches to carry out these computations can be considered. As shown by John (1953), the kinematics of fluid particles at the surface of unidirectional waves can be computed at any given location once the surface elevation and its temporal and spatial derivatives are known. While the temporal derivatives of the surface elevation $\partial \zeta / \partial t$ can be estimated from measurements, it is not so regarding $\partial \zeta / \partial x$. Moreover, in order to determine the particle path at the free surface of a water wave, the horizontal velocity everywhere at the surface should be known. This point is stressed by Bridges in his Appendix to the paper by Sclavounos (2005), where the three-dimensional generalization of the John (1953) equations was presented. The solution may also be sensitive to changes in the initial conditions that cannot be known with sufficient accuracy. Alternatively, the kinematics of the water particle at the free surface can be determined using fully nonlinear numerical models, for example such as those based on conformal mapping (Chalikov and Sheinin, 2005). Chalikov and Babanin (2012) applied this method for studies of breaking during the evolution of a random unidirectional wave field. This fully nonlinear approach does not require decomposition into separate orders.

The present study, however, is based on experiments where the shape of the steepest wave was prescribed at the leading order, and the computations of the wavemaker driving signal as well as subsequent data processing were based on the spatial version of the Zakharov (1968) equation as suggested by Kit and Shemer (2002) and Shemer et al. (2007). In order to represent faithfully the experimental conditions, the kinematics at the surface of the steep wave are computed here using the approach adopted in the derivations of the Zakharov equation.

\section{Theoretical and experimental background}

The spatial evolution of a nonlinear unidirectional wave field that takes into account third-order (quartet) interactions is written in terms of scaled amplitudes $B_{j}=B\left(\omega_{j}, x\right)$ that are related to the generalized complex "amplitudes" $a_{j}(x)=$ $a\left(\omega_{j}, x\right)$ composed of the Fourier transforms of the surface elevation $\hat{\zeta}_{j}(x)=\hat{\zeta}\left(\omega_{j}, x\right)$ and of the velocity potential at the free surface $\hat{\phi}_{j}^{\mathrm{s}}(x)=\hat{\phi}^{\mathrm{s}}\left(\omega_{j}, x\right)$ :

$a_{j}(x)=\left(\frac{g}{2 \omega_{j}}\right)^{1 / 2} \widehat{\zeta}_{j}(x)+i\left(\frac{\omega_{j}}{2 g}\right)^{1 / 2} \widehat{\phi}_{j}(x)$.

The amplitudes $a_{j}(x)$ in Eq. (2) can be seen as consisting of a sum of the free and bound waves:

$a_{j}=\left[\varepsilon B_{j}(x)+\varepsilon^{2} B_{j}^{\prime}(x)+\varepsilon^{3} B_{j}^{\prime \prime}(x)+\ldots\right] \exp \left(-i \omega_{j} t\right)$.

The amplitudes $B_{j}$ denote the so-called "free" waves that satisfy the dispersion relation for gravity waves in water of intermediate depth $h: \omega^{2}=k g \cdot \tanh (k h) ; B_{j}^{\prime}$ and $B_{j}^{\prime \prime}$ correspond to the second and third order, respectively, "bound" waves. The small parameter in the decomposition (3) $\varepsilon=k a$, where $k$ and $a$ represent the characteristic wave number and amplitude. The higher order bound components $B^{\prime}, B^{\prime \prime}$, etc. are computed for a given free wave packet $B_{j}, j=1, \ldots, N$, 
following Stiassnie and Shemer (1987); see also Krasitskii (1994). The second-order bound waves $B_{n}^{\prime}$ generated by harmonics $i$ and $j$ and their frequencies $\omega_{n}$ and wave numbers $k_{n}$ be computed using the relations

$B_{n}^{\prime(1)}=A_{n j l}^{(1)} B_{j} B_{l} ; k_{n}=k_{j}+k_{l} ; \quad \omega_{n}=\omega_{j}+\omega_{l}$,

$B_{n}^{\prime(2)}=A_{n j l}^{(2)} B_{j}^{*} B_{l} ; k_{n}=-k_{j}+k_{l} ; \omega_{n}=-\omega_{j}+\omega_{l}$,

$B_{n}^{\prime(3)}=A_{n j l}^{(3)} B_{j}^{*} B_{l}^{*} ; k_{n}=-k_{j}-k_{l} ; \omega_{n}=-\omega_{j}-\omega_{l}$.

The third-order bound waves $B_{n}^{\prime \prime}$ generated by free harmonics $i, j$, and $m$ are determined by

$$
\begin{aligned}
B_{n}^{\prime \prime(1)}= & C_{j l m}^{(1)} B_{j} B_{l} B_{m} ; \quad k_{n}=k_{j}+k_{l}+k_{m} ; \\
& \omega_{n}=\omega_{j}+\omega_{l}+\omega_{m}, \\
B_{n}^{\prime \prime(2)}= & C_{j l m}^{(2)} B_{j}^{*} B_{l} B_{m} ; \quad k_{n}=-k_{j}+k_{l}+k_{m} ; \\
& \omega_{n}=-\omega_{j}+\omega_{l}+\omega_{m}, \\
B_{n}^{\prime \prime}(3)= & C_{j l m}^{(3)} B_{j}^{*} B_{l}^{*} B_{m} ; \quad k_{n}=-k_{j}-k_{l}+k_{m} ; \\
& \omega_{n}=-\omega_{j}-\omega_{l}+\omega_{m}, \\
B_{n}^{\prime \prime}(4)= & C_{j l m}^{(4)} B_{j}^{*} B_{l}^{*} B_{m}^{*} ; k_{n}=-k_{j}-k_{l}-k_{m} ; \\
& \omega_{n}=-\omega_{j}-\omega_{l}-\omega_{m} .
\end{aligned}
$$

The coefficients $A$ in Eq. (4) and $C$ in Eq. (5) are given in the Appendix of Stiassnie and Shemer (1987). As can be seen from Eqs. (4) and (5), the phase velocities $c_{n}=\omega_{n} / k_{n}$ of higher order bound waves are not defined by the free waves' dispersion relation, but rather by frequencies and wave numbers of the "parent" free waves. The complex amplitudes $a_{j}$ that contain both free and bond waves' contributions for any prescribed wave frequency spectrum allow the computation of the temporal variation of the surface elevation

$\zeta(x, t)=\frac{1}{\pi} \operatorname{Re}\left\{\sum_{j=1}^{N} \sqrt{\frac{\omega_{j}}{2 g}} a_{j}(x) \exp \left[i\left(k_{j} x-\omega_{j} t\right)\right]\right\}$

and of the velocity potential at the free surface

$\phi^{\mathrm{s}}(x, t)=\frac{1}{\pi} \operatorname{Im}\left\{\sum_{j=1}^{N} \sqrt{\frac{g}{2 \omega_{j}}} a_{j}(x) \exp \left[i\left(k_{j} x-\omega_{j} t\right)\right]\right\}$.

The surface elevation $\zeta$, the full velocity potential $\phi$ and the horizontal $u=\partial \phi / \partial x(z=\zeta(x, t))$ and the vertical $w=$ $\partial \phi / \partial z(z=\zeta(x, t))$ velocity components at the free surface can thus be computed to the needed order.

In experiments of Shemer et al. (2002, 2007), the following Gaussian temporal variation of the surface elevation at the leading order was prescribed:

$\zeta(t)=\zeta_{0} \exp -\left(\frac{t}{m T_{0}}\right)^{2} \cos \left(\omega_{0} t\right)$ where $\omega_{0}=2 \pi / T_{0}$ is the carrier wave frequency, $\zeta_{0}$ the maximum wave amplitude in the group attained at $t=0$, and the parameter $m$ defines the width of the group. The free wave frequency spectrum of the surface elevation given by Eq. (7) is also Gaussian. Higher values of $m$ correspond to a wider group with a narrow spectrum. A decrease in $m$ renders the group narrower and the frequency spectrum wider. It was demonstrated in Shemer et al. (2007) that for $m=0.6$, the wave group in Eq. (7) contains only a single wave and thus has a wide spectrum. The small parameter representing the magnitude of nonlinearity, $\varepsilon$, is defined based on the maximum crest height $\zeta_{0}$ at the leading order and the carrier wave number, $\varepsilon=\zeta_{0} k_{0}$.

Experiments were carried out in the Large Wave Channel (GWK) in Hanover, Germany, which is $300 \mathrm{~m}$ long, $5 \mathrm{~m}$ wide, $7 \mathrm{~m}$ deep, with water depth set at $5 \mathrm{~m}$. The piston-type wavemaker was driven by a computer-generated signal that was obtained by integration of the spatial version of the $\mathrm{Za}$ kharov equation from the focusing location back to the wavemaker (see Shemer et al. 2007). The focusing location in all experiments was set at $120 \mathrm{~m}$ from the wavemaker. Two peak wave periods were used: $T_{0}=2.8 \mathrm{~s}$, corresponding to wave length of $\lambda_{0}=2 \pi / k_{0}=12.5 \mathrm{~m}$ and nearly deep water conditions $\left(k_{0} h=2.6\right)$, and $T_{0}=4.32 \mathrm{~s}\left(\lambda_{0}=25.0 \mathrm{~m}\right)$, corresponding to intermediate water depth with $k_{0} h=1.26$. The experiments were carried out for the width parameter $m=$ 0.6 at the values of the nonlinearity coefficient $\varepsilon=\zeta_{0} k_{0}$ ranging from 0.1 (corresponding to nearly linear wave regime) to those exceeding $\varepsilon=0.3$ for strongly nonlinear and breaking waves. The approach adopted in the experiments thus enabled generating either a single breaking wave or a wave on the verge of breaking at a prescribed location in the wave tank. In all experiments where breaking was observed, it occurred at the crest of the steepest wave in close vicinity of the focusing location. In particular, it was observed that the wave with $\varepsilon=0.3$ and $T_{0}=2.8 \mathrm{~s}$ does not break, while the longer wave with $T_{0}=4.34 \mathrm{~s}$ and the same steepness undergoes breaking. When the maximum steepness was reduced to $\varepsilon=0.27$ while retaining the same dominant wave period, no breaking was observed.

\section{Numerical simulations}

In view of these experimental observations, computations of the surface elevation variation with time, as well as the horizontal and vertical components of velocity and accelerations, were carried out for conditions corresponding to the focused waves in the experiments. The computations of wave shapes at the focusing location with the free wave (linear) surface elevation variation with time given by Eq. (7) were performed up to the third order in wave steepness $\varepsilon$, using Eqs. (4)-(6). The resulting contributions to the instantaneous surface elevation of the first order (proportional to $B$ ), second order $\left(\sim B^{\prime}\right)$ and third order $\left(\sim B^{\prime \prime}\right)$, as well as the total 

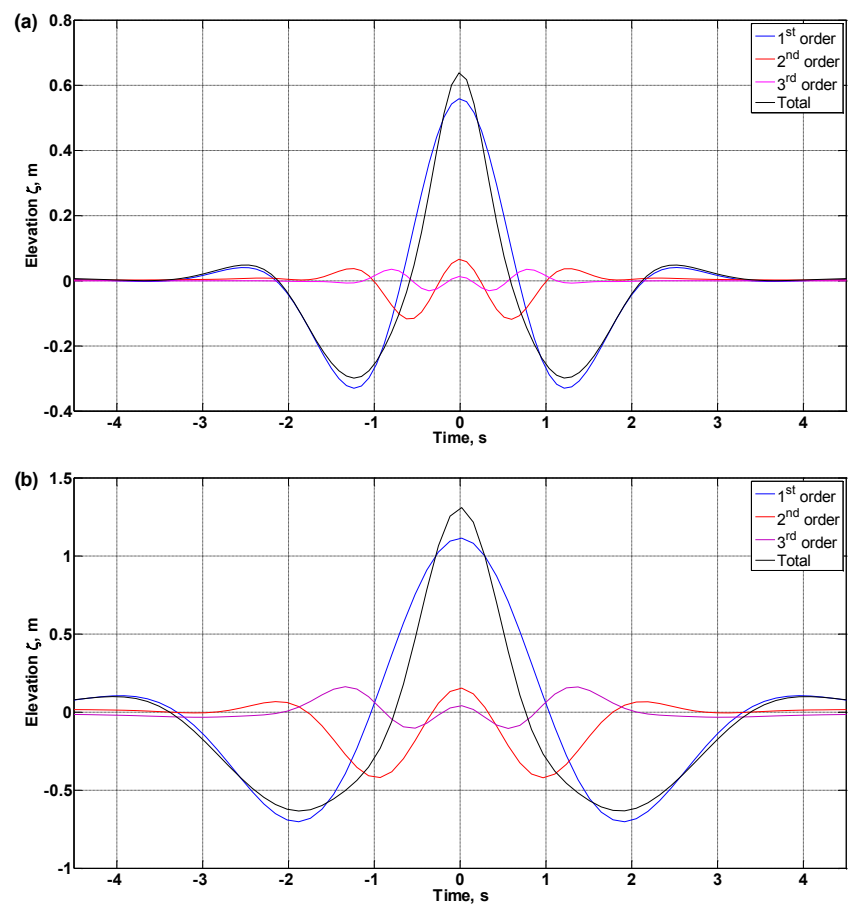

Fig. 1. Variation of the surface elevation with time for $\varepsilon=0.3$ : (a) $T_{0}=2.8 \mathrm{~s}$, dominant wave length $\lambda_{0}=12.1 \mathrm{~m}$; (b) $T_{0}=4.34 \mathrm{~s}$; $\lambda_{0}=25.0 \mathrm{~m}$.

surface elevation $\zeta(t)$ at the third order, are plotted in Fig. 1 for $\varepsilon=0.3$. The nonlinear bound waves are clearly visible in this figure; they manifest themselves in higher and sharper crests and shallower troughs of the focused wave. The effect of this variation of the wave's shape on the velocities and accelerations is now studied.

The temporal variation of two components of water velocity at the focusing location is plotted in Fig. 2. The contributions to the total horizontal velocity at the crest $(t=0)$ at all orders are positive; as expected, all vertical velocity contributions at the crest vanish. Higher order contributions to horizontal velocity are relatively more significant than those for the wave shape in Fig. 1. Note also that for the conditions of Fig. 2, the third-order contribution to the horizontal velocity $u$ in the vicinity of the crest is not very different from that at the second order. For longer dominant wave period, $T_{0}=4.34 \mathrm{~s}$, corresponding to effectively shallower water, and the same steepness of $\varepsilon=0.3$, the temporal variation of both velocity components at all orders is qualitatively similar to that of Fig. 2 and for that reason not presented here. In this case, the contributions of the second and third order to the horizontal velocity at the crest are practically equal and even more important, each contributing to the total horizontal velocity at the crest more than $25 \%$ of the leading order part. For a wave group in water of intermediate depth, the extreme value of the horizontal velocity is thus increased by more than $50 \%$ as compared to the linear solution.
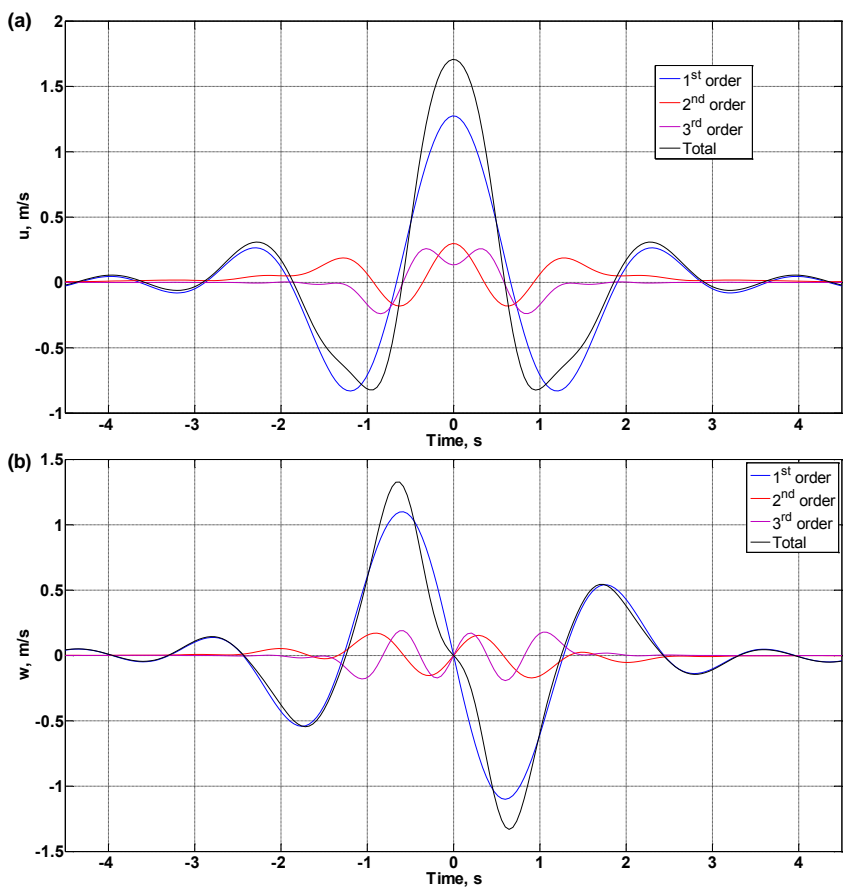

Fig. 2. Surface velocity at the focusing location for $\varepsilon=0.3$ and $T_{0}=2.8 \mathrm{~s}$ : (a) horizontal component $u$; (b) vertical component $w$.

The computed horizontal velocities at the wave crest for the nonlinearity parameter $\varepsilon=0.3$ are $u_{\max }=1.69 \mathrm{~m} \mathrm{~s}^{-1}$ for the wave group with shorter dominant wave, $T_{0}=2.8 \mathrm{~s}$, and $u_{\max }=3.09 \mathrm{~m} \mathrm{~s}^{-1}$ for $T_{0}=4.34 \mathrm{~s}$. These results can be compared with the corresponding wave propagation velocities. For relatively deep water case of $T_{0}=2.8 \mathrm{~s}$, the dominant wave's phase velocity according to the linear dispersion relation is $c_{\mathrm{p}}=4.32 \mathrm{~m} \mathrm{~s}^{-1}$, and the group velocity only slightly exceeds the deep water relation $c_{\mathrm{g}}=c_{\mathrm{p}} / 2$; the calculated for this wave period $c_{\mathrm{g}}=2.29 \mathrm{~m} \mathrm{~s}^{-1}$. The corresponding values of the longer wave $\left(T_{0}=4.34 \mathrm{~s}\right)$ are $c_{\mathrm{p}}=5.76 \mathrm{~m} \mathrm{~s}^{-1}$ and $c_{\mathrm{g}}=4.06 \mathrm{~m} \mathrm{~s}^{-1}$. The phase and group velocities, however, can serve as characterizing parameters only for wave groups with narrow spectra. As stressed in Shemer et al. (2007), appearance of extremely steep waves by focusing mechanism is a result of constructive interference of numerous harmonics and thus requires a broad spectrum. Therefore both phase $c_{\mathrm{p}}$ and group $c_{\mathrm{g}}$ velocities calculated for the dominant wave are not directly related to the actual crest propagation velocities $u_{\mathrm{cr}}$. As can be seen from Fig. 1, the higher order contributions do not affect the instant at which the steepest wave appears. The velocity of the crest was therefore determined by computing the instantaneous wave forms at several instants in the vicinity of the focusing at $t=0$ at the leading order yielding $c_{\mathrm{cr}}=3.6 \mathrm{~m} \mathrm{~s}^{-1}$ for $T_{0}=2.8 \mathrm{~s}$ and $c_{\mathrm{cr}}=5.0 \mathrm{~m} \mathrm{~s}^{-1}$ for $T_{0}=4.34 \mathrm{~s}$. In both these cases the crest velocity $c_{\mathrm{cr}}$ of the broad-spectrum wave group is significantly different from both the phase $c_{\mathrm{p}}$ and the group $c_{\mathrm{g}}$ velocities of the 
dominant wave and satisfies the condition $c_{\mathrm{g}}<c_{\mathrm{cr}}<c_{\mathrm{p}}$. For the dominant wave lengths considered, the maximum velocities at the crest containing terms up to the third order are significantly lower than $c_{\mathrm{cr}}$ :

$u_{\max } / c_{\mathrm{cr}}=0.47$ for $T_{0}=2.8 \mathrm{~s}$

and

$u_{\max } / c_{\mathrm{cr}}=0.62$ for shallower water with $T_{0}=4.34 \mathrm{~s}$.

Vertical accelerations are considered next. Since the emphasis of this study is on nonlinear effects that are more pronounced in intermediate depth, the results corresponding to the longer wave case are mainly presented in sequel. It is instructive to compare different relevant accelerations quantitatively. The contributions at different orders to the "apparent" vertical acceleration $\partial^{2} \zeta / \partial t^{2}$ that can be relatively easily estimated in experiments from the data recorded by a fixed wave gauge are plotted in Fig. 3a. A similar plot of the Eulerian vertical acceleration component $a_{\mathrm{E}}=\partial w / \partial t$ is presented in Fig. 3b. In the linear approximation these two accelerations are indeed identical. Contributions at higher orders, however, are quite different in Fig. 3a and b and are comparable with the linear term, in particular in Fig. 3a. The importance of higher order terms in computations of acceleration stems from the more significant role of the high frequency part of the spectrum as compared to the total velocity (see Fig. 2). The "apparent" and the Eulerian accelerations accurate to the third order differ considerably, both qualitatively and quantitatively. The negative "apparent" acceleration at the wave crest exceeds $8 \mathrm{~m} \mathrm{~s}^{-2}$, quite close to the acceleration of gravity $g$. The value of $a_{\mathrm{E}}$ in Fig. $3 \mathrm{~b}$ is only about $-5.5 \mathrm{~m} \mathrm{~s}^{-2}$ at this instant; it should be stressed though that it still exceeds $g / 2$.

As stressed above, the physical reasoning behind the dynamic criterion by Phillips implies that the negative vertical acceleration of any water particle cannot exceed $g$. To verify whether this criterion is applicable for the experimental conditions considered, Lagrangian acceleration $a_{\mathrm{L}}$ has to be calculated. For unidirectional waves, $a_{\mathrm{L}}$ is a sum of $a_{\mathrm{E}}$ and the convective acceleration:

$a_{\mathrm{L}}=D w / D t=\partial w / \partial t+u \cdot \partial w / \partial x+w \cdot \partial w / \partial z$.

The convective acceleration only contributes at the second order and higher. To compute the corresponding terms with the third-order accuracy, it is sufficient to calculate both velocity components, and their spatial derivatives have up to the second order. The convective acceleration terms in Eq. (8) at the second and the third order are plotted in Fig. 4. The indices in the legends denote the order of the corresponding terms. It is obvious that in general, in spite of the fact that the convective acceleration is a quantity of higher order, for steep waves it cannot be disregarded. Although the contribution of the term $w \cdot \partial w / \partial z$ at the wave crest is zero at all orders (see Fig. 4b) since the vertical velocity vanishes at $t=0$, this term
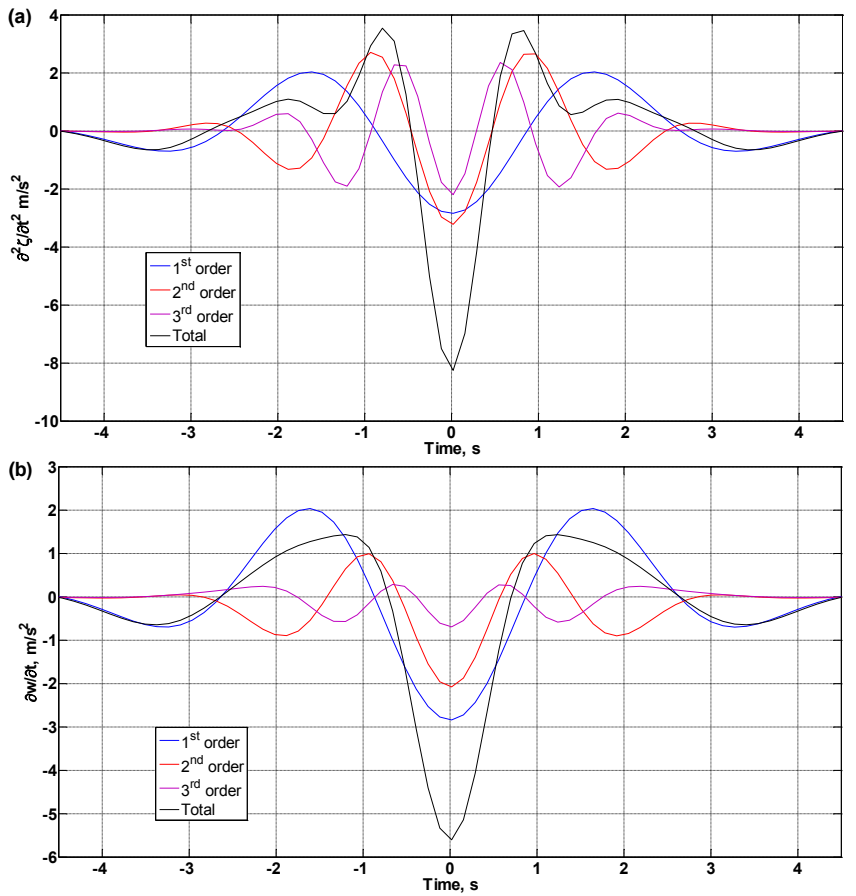

Fig. 3. (a) The "apparent" vertical acceleration $\partial^{2} \zeta / \partial t^{2}$ and (b) the Eulerian vertical acceleration $\partial w / \partial t$ for $\varepsilon=0.3, T_{0}=4.34 \mathrm{~s}$; $\lambda_{0}=25.0 \mathrm{~m}$.
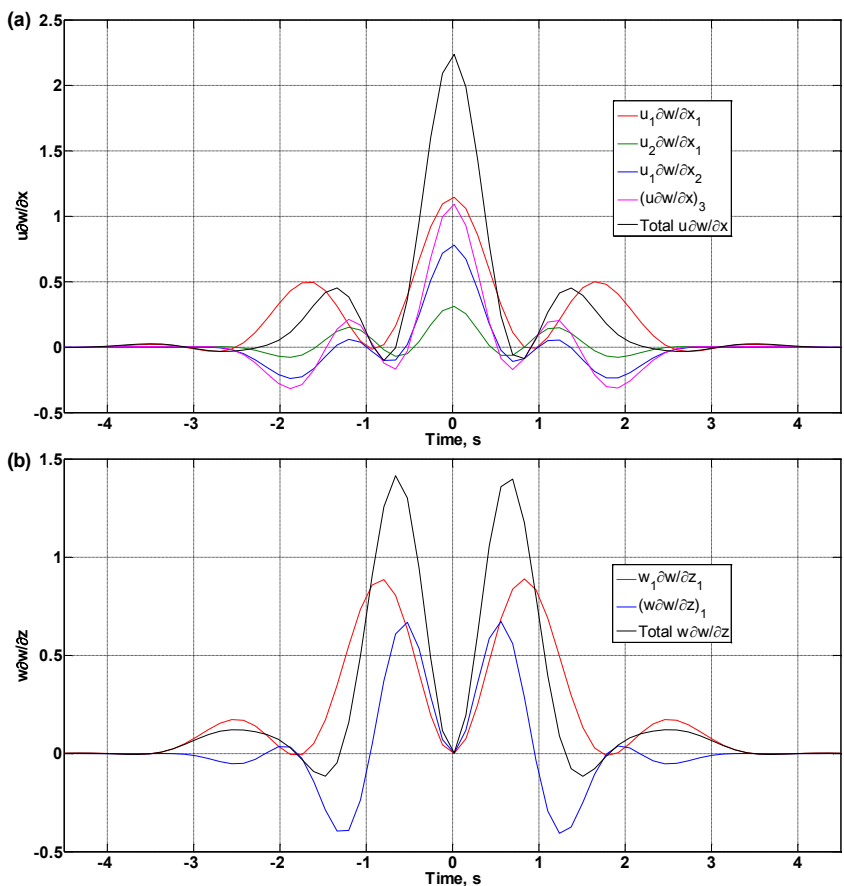

Fig. 4. Terms contribution to the convective vertical acceleration for the conditions of Fig. 3: (a) $u \cdot \partial w / \partial x$; (b) $w \cdot \partial w / \partial z$.

may become quite larger at other instants. Even more important for the present discussion is the fact that at $t=0$ the contributions at all terms to $u \cdot \partial w / \partial x$ in Fig. 4 a are positive and 
relatively large. The essentially positive convectional acceleration at the wave crest therefore reduces considerably the maximum value of the negative vertical acceleration as compared to $a_{\mathrm{E}}$. It is also worth noting that at $t=0$, the contribution of the second-order term $u_{1}(\partial w / \partial x)_{1}$ is nearly identical to the total third-order term $u_{1}(\partial w / \partial x)_{2}+u_{2}(\partial w / \partial x)_{1}$.

The computed Lagrangian accelerations at the focusing location are plotted in Fig. 5 for both wave periods in the experiments. The extreme negative value of the vertical component of the Lagrangian acceleration attained at the wave crest accurate to the third order in both cases is close to about $g / 3$, well below the value predicted by the Phillips dynamic criterion. Although higher order corrections are essential in computations of the "apparent", Eulerian and convective accelerations (see Figs. 3 and 4), their respective contributions effectively cancel out in computation of the Lagrangian acceleration, so that the resulting temporal variation of $a_{\mathrm{L}}$ does not differ substantially from the linear solution. A qualitative and relatively minor difference can be noticed between the accelerations in the vicinity of the crest in Fig. 5a and b. For $k_{0} h=2.6$ in Fig. 5a, the total negative Lagrangian acceleration at $t=0$ is smaller than the linear value, while for shallower water with $k_{0} h=1.26$ in Fig. $5 \mathrm{~b}$ the opposite is correct. This dissimilarity can be attributed to the increasing contribution of higher order bound waves with decreasing dimensionless depth $k_{0} h$ (cf. Fig. 2a and b).

\section{Discussion and conclusions}

In the present study an attempt is made to relate the kinematics of a wave group to experimentally observed wave breaking events in a tank under controlled conditions (Shemer et al. 2007). For wave steepness of $\varepsilon=0.3$, the steepest wave in a focused wave group with a wide Gaussian-shaped spectrum wave (at the leading order) was observed to be on the verge of breaking for the shorter dominant wave length corresponding to $k_{0} h=2.6$, and actually underwent breaking for a longer wave with $k_{0} h=1.26$. For the temporal variation of the surface elevation corresponding to that in the experiments at the focusing location, velocities and accelerations at the surface are computed to the third order in $\varepsilon$. The analysis is based on the Zakharov (1968) equation. The various orders' contributions to the surface elevation as well as to the horizontal and vertical velocity components are accounted for. In general, the orbital velocities computed at the crest of steep waves close to breaking are in agreement with recent direct measurements by Grue and Jensen (2012).

In an effort to verify the applicability of the Phillips (1958) dynamic criterion for wave breaking, particular attention is given in the present study to computations of the vertical acceleration. Clear distinction is made between the "apparent", Eulerian $\left(a_{\mathrm{E}}\right)$ and Lagrangian $\left(a_{\mathrm{L}}\right)$ accelerations. The time dependence of all those accelerations for the focused wave was obtained. It is shown that the extreme negative va-
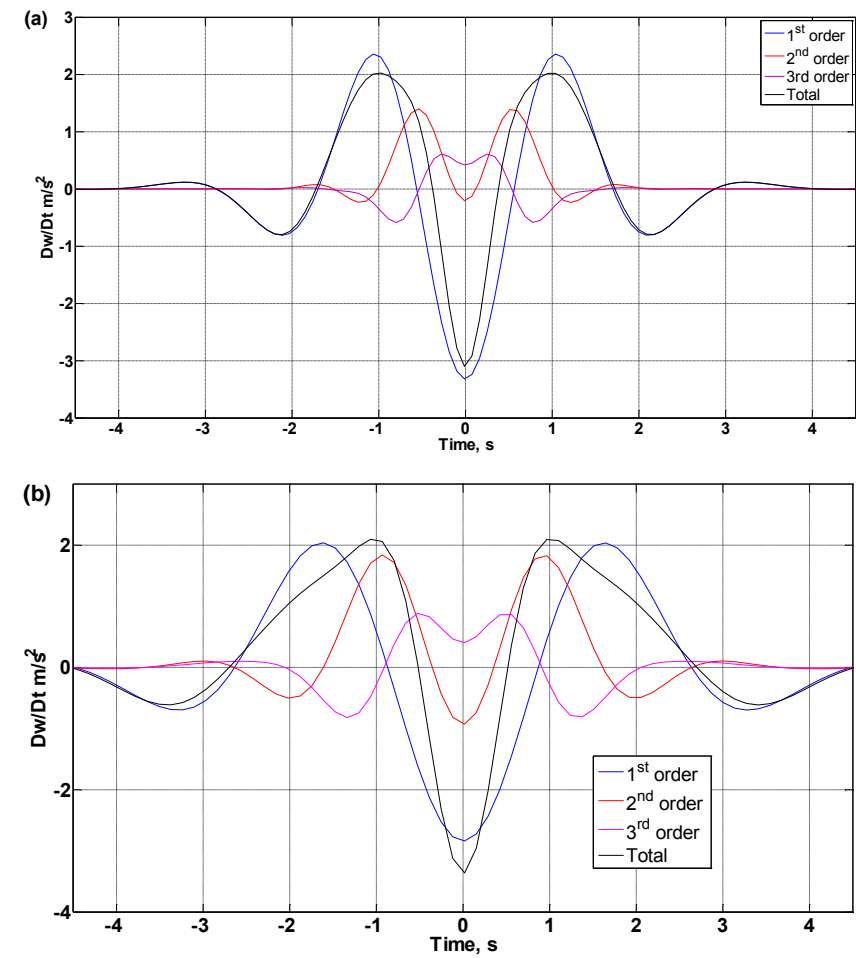

Fig. 5. Lagrangian vertical acceleration $a_{\mathrm{L}}$ at the focusing location for $\varepsilon=0.3$ : (a) $T_{0}=2.8 \mathrm{~s}, \lambda_{0}=12.1 \mathrm{~m}$; (b) $T_{0}=4.34 \mathrm{~s} ; \lambda_{0}=$ $25.0 \mathrm{~m}$.

lues of the "apparent" acceleration $\partial^{2} \zeta / \partial t^{2}$ with nonlinear terms accounted for indeed exceed significantly those computed in the linear approximation. The same is true regarding the Eulerian vertical acceleration $a_{\mathrm{E}}=\partial w / \partial t$, although the extreme negative values of $a_{\mathrm{E}}$ are somewhat smaller than those of $\partial^{2} \zeta / \partial t^{2}$. The convective acceleration terms are, however, mostly positive and thus lead to even smaller negative Lagrangian acceleration $a_{\mathrm{L}}$ at the crest of a steep wave. It was observed that for the cases considered, even the value of the "apparent" negative acceleration $\partial^{2} \zeta / \partial t^{2}$ at the crest of the steepest possible wave at this order remains somewhat smaller than $g$. The nonlinear contributions decrease the extreme values of negative Eulerian and Lagrangian vertical accelerations as compared to $\partial^{2} \zeta / \partial t^{2}$.

The Lagrangian acceleration is of particular importance since it is the appropriate quantity in the Phillips dynamic breaking criterion. The present results show that the extreme values of $a_{\mathrm{L}}$ for waves on the verge of breaking remain well below the gravity acceleration $g$. It appears that virtually all nonlinear additions to the maximum apparent negative vertical acceleration are effectively cancelled out by the positive convective acceleration, so that the maximum negative values of the Lagrangian acceleration accurate to the third order in the wave steepness $\varepsilon$ are in fact quite close to the vertical acceleration calculated in the linear approximation. This observation makes it plausible to assume that extension 
of computations to even higher order will not change significantly the resulting vertical component of $a_{\mathrm{L}}$. It thus appears that the Phillips criterion cannot serve for prediction of wave breaking only for Stokes waves, as demonstrated by Longuet-Higgins (1985), or for more realistic wide-spectra nonlinear wave groups.

This failure of the dynamic criterion prompts a closer look at the kinematic wave breaking criteria that relate the water velocities at the surface with wave propagation velocities. The kinematic condition states that a wave breaks when the water particle velocity at the crest of the wave exceeds the crest velocity, which is often represented by the phase velocity $c_{\mathrm{p}}$ at the dominant wave frequency. Alternatively, since the envelope of a narrow-banded group propagates with the group velocity $c_{\mathrm{g}}$ of the peak wave, the value of $c_{\mathrm{g}}$ sometimes is taken as the characteristic wave velocity. It is demonstrated here, however, that for a wider spectrum, neither $c_{\mathrm{p}}$ nor $c_{\mathrm{g}}$ corresponds to the propagation velocity of the highest wave crest. The actual velocity of crest that should be used in the analysis of breaking conditions for waves with wider spectra therefore has to be either computed directly for any given complex wave spectrum or determined experimentally.

The horizontal components of orbital velocity computed in the present study at the crest $u_{\max }$ accurate to the third order remain notably smaller than the estimated corresponding propagation velocities of crests of extreme waves, thus suggesting that the kinematic condition is also not satisfied for wave parameters considered here. Nevertheless, it should be stressed that contribution of the third-order terms to $u_{\max }$ is relatively large and comparable to the contribution to the maximum horizontal velocity at the crest at the second order. Either application of fully nonlinear methods as those reviewed in the Introduction or extension of computations to higher order is thus needed to determine the applicability of the kinetic criterion for wave breaking. Alternatively, direct measurements of water particle velocities at the surface in the vicinity of the crest of a breaking wave should be carried out.

Acknowledgements. This work was supported by a grant \# 2010219 from US-Israel Binational Science Foundation.

Edited by: A. Slunyaev

Reviewed by: two anonymous referees

\section{References}

Babanin, A.: Breaking and dissipation of ocean surface waves, Cambridge University Press, Cambridge, UK, 2011.

Chalikov, D. and Babanin, A. V.: Simulation of wave breaking in one-dimensional spectral environment, J. Phys. Oceanogr., 42, 1745-1761, doi:10.1175/JPO-D-11-0128.1, 2012.

Chalikov, D. and Sheinin, D: Modeling of extreme waves based on equations of potential flow with a free surface, J. Comput. Phys., 210, 247-273, doi:10.1016/j.jcp.2005.04.008, 2005.
Gemmrich, J.: On occurence of wave breaking, in: Proc. Rogue waves: "Aha Huliko" Hawaiian winter workshop, Univ. Hawaii at Manoa, Honolulu, 123-130, 2005.

Grue, J. and Jensen, A.: Orbital velocity and breaking in steep random waves, J. Geophys. Res., 117, C07013, doi:10.1029/2012JC008024, 2012.

Jensen, A., Clamond, D., Huseby, M., and Grue, J.: On local and convective accelerations in steep wave events, Ocean Eng., 34, 426-435, doi:10.1016/j.oceaneng.2006.03.013, 2007.

Johannessen, T. B.: Calculations of kinematics underneath measured time histories of steep water waves, Appl. Ocean Res., 32, 391-403, doi:10.1016/j.apor.2010.08.002, 2010.

John, F.: Two-dimensional potential flow with a free boundary, Commun. Pur. Appl. Math., 6, 497-503, doi:10.1002/cpa.3160060405, 1953.

Kit, E. and Shemer, L.: Spatial versions of the Zakharov and Dysthe evolution equations for deep-water gravity waves, J. Fluid Mech., 450, 201-205, doi:10.1017/S0022112001006498, 2002.

Krasitskii, V. P.: On reduced equations in the Hamiltonian theory of weakly nonlinear surface waves, J. Fluid Mech., 272, 1-20, doi:10.1017/S0022112094004350, 1994.

Longuet-Higgins, M. S.: Accelerations in steep gravity waves, J. Phys. Oceanogr., 15, 1570-1579, doi:10.1175/15200485(1985)015<1570:AISGW>2.0.CO;2, 1985.

Longuet-Higgins, M. S. and Fox, M. G. H: Theory of the almost highest wave: The inner solution, J. Fluid Mech., 80, 721-741, doi:10.1017/S0022112077002444, 1977.

Phillips, O. M.: The equilibrium range in the spectrum of wind generated waves, J. Fluid Mech., 4, 785-790, doi:10.1017/S0022112058000550, 1958.

Sclavounos, P. D.: Nonlinear particle kinematics of ocean waves, J. Fluid Mech., 540, 133-142, doi:10.1017/S0022112005004878, 2005.

Shemer, L., Kit, Y., and Jiao, H.-Y.: An experimental and numerical study of the spatial evolution of unidirectional nonlinear water-wave groups, Phys. Fluids, 17, 3380-3390, doi:10.1063/1.1501908, 2002.

Shemer, L., Goulitski, K., and Kit, E.: Evolution of widespectrum wave groups in a tank: an experimental and numerical study, Eur. J. Mech. B-Fluid., 26, 193-219, doi:10.1016/j.euromechflu.2006.06.004, 2007.

Shemer, L., Sergeeva, A., and Liberzon, D.: Effect of the initial spectrum on the spatial evolution of statistics of unidirectional nonlinear random waves, J. Geophys. Res., 115, C12039, doi:10.1029/2010JC006326, 2010.

Stiassnie, M. and Shemer, L.: Energy computations for coupled evolution of Class I and Class II instabilities of Stokes waves, J. Fluid Mech., 174, 299-312, doi:10.1017/S0022112087000132, 1987.

Zakharov, V. E.: Stability of periodic waves of finite amplitude on the surface of a deep fluid, J. Appl. Mech. Tech. Phy., 9, 190-194, doi:10.1007/BF00913182, 1968. 